\title{
The anti-obesity drug orlistat promotes sensitivity to TRAIL by two different pathways in hormone-refractory prostate cancer cells
}

\author{
JUN FUJIWARA ${ }^{1,2}$, YOSHIHIRO SOWA ${ }^{1}$, MANO HORINAKA ${ }^{1}$, MAKOTO KOYAMA ${ }^{1}$, \\ MIKI WAKADA $^{1}$, TSUNEHARU MIKI ${ }^{2}$ and TOSHIYUKI SAKAI ${ }^{1}$ \\ ${ }^{1}$ Department of Molecular-Targeting Cancer Prevention, Graduate School of Medical Science, ${ }^{2}$ Department of Urology, \\ Kyoto Prefectural University of Medicine, Kawaramachi-Hirokoji, Kamigyo-ku, Kyoto 602-8566, Japan
}

Received October 21, 2011; Accepted December 14, 2011

DOI: $10.3892 /$ ijo.2012.1353

\begin{abstract}
The administration of tumor necrosis factor-related apoptosis-inducing ligand (TRAIL) is one of the expected cancer therapeutics. However, improvements are required in therapies against TRAIL-resistant tumor cells. We report, here, that the anti-obesity drug orlistat enhances the sensitivity to TRAIL in hormone-refractory prostate cancer (HRPC) cells through two different pathways. The combination of orlistat and TRAIL remarkably induced apoptosis in TRAIL-resistant HRPC, DU145 and PC3 cells. Orlistat induced the expression of death receptor (DR) 5 , which is one of the TRAIL receptors, at both the mRNA and protein levels. The suppression of DR5 with siRNA reduced the apoptosis induced by the combination of orlistat and TRAIL, suggesting that the apoptosis was at least partially due to the upregulation of DR5. Although the upregulation by orlistat of CHOP at both mRNA and protein levels was observed in both cell lines, the activation of the DR5 promoter in DU145 cells was CHOP-dependent, but that in PC3 cells was CHOP-independent. Moreover, orlistat induced reactive oxygen species (ROS), and a ROS scavenger diminished the sensitivity to TRAIL through the suppression of CHOP and DR5 expression in both cell lines. These results suggest that there are two pathways of upregulation of DR5 by orlistat, which are the ROS-CHOP pathway and the ROS-direct pathway. In conclusion, orlistat promotes the sensitivity to TRAIL by ROS-mediated pathways in prostate cancer cells, especially in TRAIL-resistant cells. We believe that the combination of orlistat and TRAIL in HRPC is promising as a new chemotherapeutic strategy.
\end{abstract}

Correspondence to: Dr Yoshihiro Sowa, Department of MolecularTargeting Cancer Prevention, Graduate School of Medical Science, Kyoto Prefectural University of Medicine, Kawaramachi-Hirokoji, Kamigyo-ku, Kyoto 602-8566, Japan

E-mail: ysowa@koto.kpu-m.ac.jp

Key words: orlistat, tumor necrosis factor-related apoptosis-inducing ligand, prostate cancer, reactive oxygen species

\section{Introduction}

It has been reported that several anti-hyperlipidemia medicines unexpectedly exert anti-tumor effects (1-3). Among these drugs, orlistat $\left(\mathrm{Xenical}^{\mathrm{TM}}\right)$ is well known as an anti-obesity agent, which inhibits lipase activity by direct binding, and has been used for more than a decade in the US and Europe. Recently, it was reported that orlistat also binds to fatty acid synthase (FAS) and inhibits its activity (4). The expression of FAS was upregulated in many malignant tumors, and FAS inhibitors, cerulenin, c75 and orlistat, induced apoptosis and cell cycle arrest in prostate and breast cancer cell lines (4-7). However, the precise molecular mechanisms of the anti-tumor effects of orlistat are still unclear.

Tumor necrosis factor-related apoptosis-inducing ligand (TRAIL) selectively induces apoptosis in various malignant tumor cells in vitro and in vivo, with little or no toxicity in normal cells and tissues $(8,9)$. Endogenous TRAIL plays a critical role in immune-surveillance against tumor development (10). Death receptor 5 (DR5) is one of the cell surface receptors for TRAIL, and several studies have reported that DR5 is expressed more abundantly in cancer cells than in normal cells $(11,12)$. Therefore, both the administration of TRAIL and the enhancement of TRAIL expression are considered promising strategies for cancer therapy and prevention. However, since some tumors are resistant to TRAIL-induced apoptosis (13), we need to overcome TRAIL-resistance in cancer cells.

Reactive oxygen species (ROS) are known to be important for both carcinogenesis and chemotherapy. In malignant tumor cells, several anti-tumor agents and radiation therapy induce ROS and ER stress, subsequently causing apoptosis (14-16). In the present study, we found that orlistat upregulated DR5 expression by the ROS induction, and subsequently enhanced the sensitivity to TRAIL in hormone-refractory prostate cancer (HRPC) cells but not in normal cells.

\section{Materials and methods}

Cell culture. Human prostate cancer cell lines PC3 and DU145 were maintained in RPMI-1640 with $10 \%$ fetal bovine serum, $2 \mathrm{mM} \mathrm{L}$-glutamine, $100 \mathrm{U} / \mathrm{ml}$ penicillin, and $100 \mu \mathrm{g} / \mathrm{ml}$ 
(a)

DU145

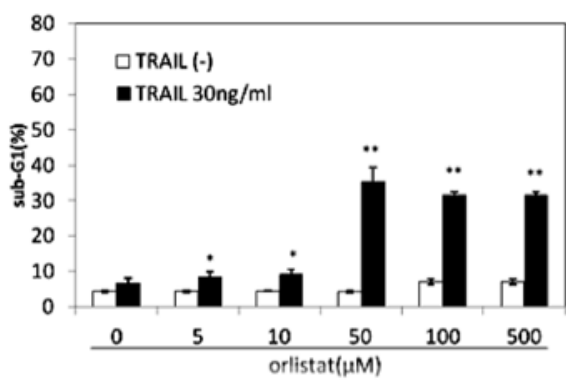

(b)

DU145

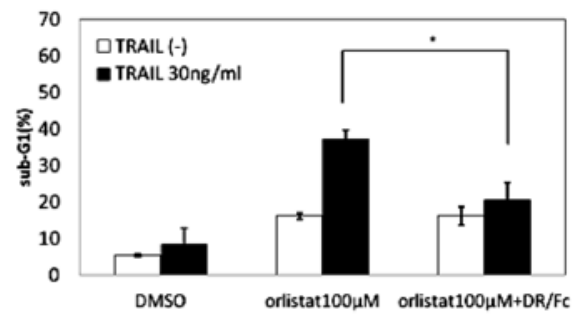

(c)

DU145

\begin{tabular}{|c|c|c|c|}
\hline orlistat $(\mu \mathrm{M})$ & TRAIL(ng/ml) & $\begin{array}{c}\text { Fractional } \\
\text { effect }\end{array}$ & $\mathbf{~ C l}$ \\
\hline 25 & 15 & 0.531 & $\mathbf{0 . 1 4 6}$ \\
\hline 50 & 30 & 0.587 & $\mathbf{0 . 1 6 5}$ \\
\hline 100 & 60 & 0.624 & $\mathbf{0 . 2 8}$ \\
\hline 200 & 120 & 0.639 & $\mathbf{0 . 5 2 2}$ \\
\hline
\end{tabular}

PC3

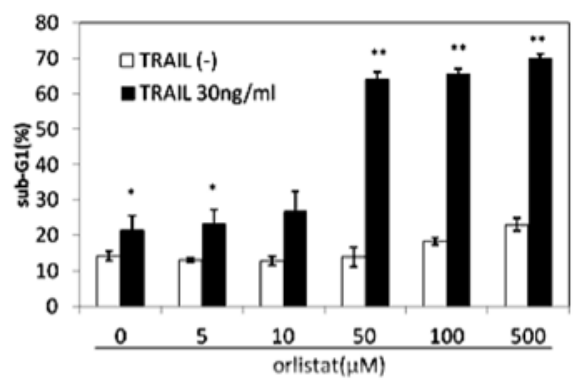

PC3

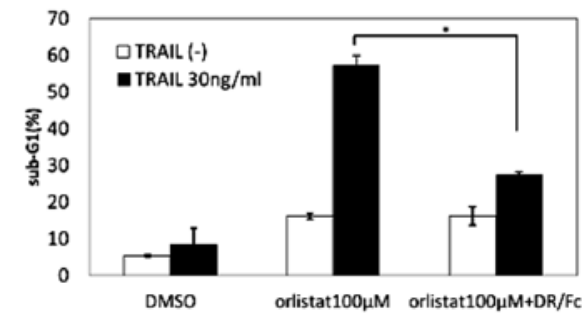

PC3

\begin{tabular}{|c|c|c|c|}
\hline orlistat $(\mu \mathrm{M})$ & TRAIL(ng/ml) & $\begin{array}{c}\text { Fractional } \\
\text { effect }\end{array}$ & Cl \\
\hline 25 & 15 & 0.454 & 0.068 \\
\hline 50 & 30 & 0.61 & 0.092 \\
\hline 100 & 60 & 0.667 & $\mathbf{0 . 1 4}$ \\
\hline 200 & 120 & 0.711 & $\mathbf{0 . 2 6 1}$ \\
\hline
\end{tabular}

Figure 1. Orlistat promotes TRAIL sensitivity in DU145 and PC3 cells. (a) DU145 or PC3 cells were treated with 30 ng/ml of TRAIL and/or various concentrations of orlistat for $24 \mathrm{~h}$. The population of apoptotic cells was analyzed as described in Materials and methods. Columns, mean ( $\mathrm{n}=3$ ); bars, $\mathrm{SD}$. ${ }^{*} \mathrm{P}<0.05,{ }^{* *} \mathrm{P}<0.01$ compared with the TRAIL (-). (b) DU145 or PC 3 cells were treated with $30 \mathrm{ng} / \mathrm{ml}$ TRAIL and/or $100 \mu \mathrm{M}$ orlistat with or without $1 \mu \mathrm{g} / \mathrm{ml}$ DR5/Fc chimeric protein for $24 \mathrm{~h}$. The population of apoptotic cells was analyzed. Columns, mean ( $=3)$; bars, SD. ${ }^{*} \mathrm{P}<0.05$ compared with TRAIL (-). (c) The combination index (CI) of TRAIL and orlistat was calculated in DU145 or PC3 cells.

streptomycin. Normal peripheral blood mononuclear cells (PBMC) were isolated by Ficoll-Paque PLUS (GE Healthcare, Waukesha, WI) according to the manufacturer's instructions and maintained in X-VIVO 20 (Cambrex, Charles City, IA) serum-free medium with $0.2 \%$ bovine serum albumin (SigmaAldrich, St. Louis, MO). Normal human prostate epithelial cells (PrEC) were obtained from Cell Systems (Kirkland, WA) and maintained in SFM CS-C medium (Cell Systems) according to the instructions of the supplier. All cells were incubated at $37^{\circ} \mathrm{C}$, in a humid atmosphere with $5 \% \mathrm{CO}_{2}$.

Reagents. Orlistat and catalase were purchased from SigmaAldrich. Soluble recombinant human TRAIL/Apo2L (untagged TRAIL, amino acids 114-281) was purchased from PeproTech (Rocky Hill, NJ). Human recombinant DR5/Fc chimera was purchased from R\&D Systems (Minneapolis, MN).

Detection of apoptosis. DNA fragmentation as a marker of apoptosis was quantified with the hypodiploid DNA (sub- $\mathrm{G}_{1}$ ) population. In brief, cells were seeded at $1.0 \times 10^{4}$ per well in 24-well culture plates and incubated for $24 \mathrm{~h}$. The cells were then exposed to the indicated concentrations of orlistat, and the sub- $\mathrm{G}_{1}$ population was analyzed using a Guava EasyCyte plus flow cytometer according to the manufacturer's instructions (Millipore, Billerica, MA).

Plasmid preparation. As previously described (17), the digested SacI-NcoI fragment from the DR5 promoter region of human genomic DNA was subcloned into the SacI-NcoI site of the pGVB2 luciferase assay vector (Toyo Ink, Tokyo, Japan) to produce pDR5PF. Deletion and point-mutated mutants of pDR5PF, termed pDR5/-347, pDR5/-252 and pDR5/ mtCHOP with a mutation in the CHOP-binding site at -272 / -269 , were generated as previously reported (18). Full-length CHOP promoter, $\mathrm{pCHOP}$, deletion mutant, $\mathrm{pCHOP} / 150$, and point-mutated mutant, $\mathrm{pCHOP}$-ERSE with mutation in the endoplasmic reticulum stress responsive element (ERSE), were generated as previously reported (19).

Transfection and luciferase assay. A series of DR5 and CHOP reporter plasmids and a vacant vector plasmid $(1.0 \mu \mathrm{g})$ were transfected into the cells $\left(1.0 \times 10^{5}\right.$ cells/well) using the DEAE-dextran 
(a)
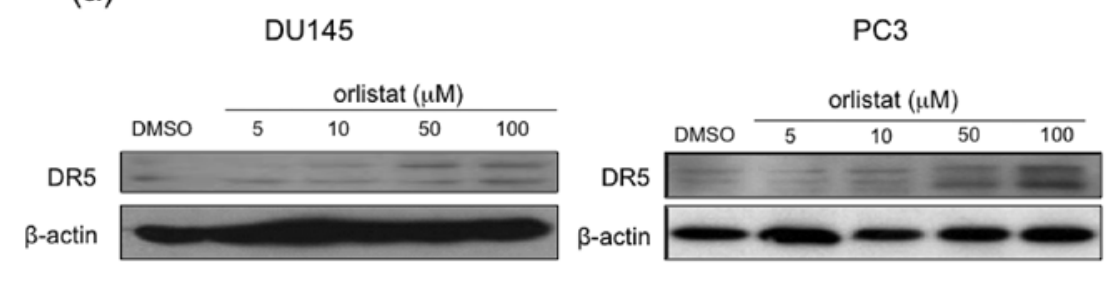

(b)

DU145

PC3
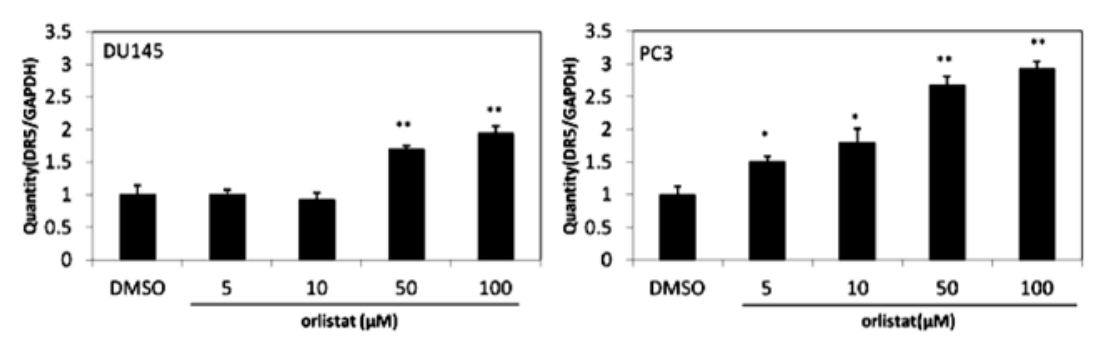

Figure 2. Orlistat induces DR5 protein and mRNA expression. (a) Whole cell lysates of DU145 or PC3 were prepared with various concentrations of orlistat for $24 \mathrm{~h}$. The lysates were analyzed by western blotting for DR5 expression. $\beta$-actin was used as a loading control. (b) The induction of DR5 mRNA by orlistat in DU145 or PC3 cells was analyzed by real-time RT-PCR as described in Materials and methods. Columns, mean ( $\mathrm{n}=3$ ); bars, $\mathrm{SD}$. ${ }^{*} \mathrm{P}<0.05$, ${ }^{* *} \mathrm{P}<0.01$ compared with the DMSO control. Each sample was standardized by the expression of glyceraldehyde-3-phosphate (GAPDH) as an internal control.

method (GE Healthcare). After $24 \mathrm{~h}$, the cells were treated with or without orlistat $(100 \mu \mathrm{M})$ for $24 \mathrm{~h}$ and then harvested. Luciferase activity was measured in triplicate, and the experiments were repeated several times.

RNA isolation and quantification analysis by real-time RT-PCR. The DR5 mRNA expression was determined by reverse transcription-PCR (RT-PCR). Total cellular RNA was extracted from cells using the Qiagen-RNeasy Mini kit (Qiagen, Hilden, Germany), and cDNA was synthesized from $2 \mathrm{mg}$ of total RNA using M-MLV reverse transcriptase (Promega, Madison, WI) and amplified by PCR using Taqman probes purchased from Applied Biosystems (Carlsbad, CA). Real-time RT-PCR was performed as previously described (18).

Western blot analysis. Whole cell lysate containing $50 \mu \mathrm{g}$ of protein was separated on a 10 or $12.5 \%$ SDS-polyacrylamide gel for electrophoresis and blotted onto polyvinylidene difluoride membranes (Millipore). Human DR5 (ProSci, Poway, CA), CHOP (GADD153; Sigma-Aldrich) and $\beta$-actin (Sigma-Aldrich) antibodies were used as the primary antibodies. The signal was detected with an enhanced chemiluminescence western blot analysis system (GE Healthcare).

Small interfering RNAs. The DR5, CHOP, and non-targeting siRNA were synthesized by Sigma-Aldrich $(18,20)$. Before transfection, the cells were seeded into the medium without antibiotics at a density of 30-40\%. The siRNA was transfected by oligofectamine (Invitrogen, Carlsbad, CA). After $24 \mathrm{~h}$ of transfection, the cells were treated with orlistat and/or TRAIL and then harvested.

Measurement of intercellular ROS. Cells were pretreated with $100 \mu \mathrm{M}$ orlistat or DMSO for $12 \mathrm{~h}$ and then treated with $10 \mu \mathrm{M}$ 5-(and-6)-chloromethyl-2,7-dichlorodihydrofluorescein diacetate, acetyl ester (CM-H ${ }_{2}$ DCFDA) (Molecular Probes, Carlsbad,
CA). After 30 min of incubation with $\mathrm{CM}-\mathrm{H}_{2} \mathrm{DCFDA}$, the fluorescence was measured by flow cytometry. The geo-mean fluorescence intensity at $530 \mathrm{~nm}$ was calculated.

Statistical analysis. Statistical analysis was performed using a two-tailed, paired Student's t test. Samples were considered significantly different at $\mathrm{P}<0.05$.

\section{Results}

Orlistat promotes the TRAIL sensitivity in prostate cancer DU145 and PC3 cells. We investigated the effects of orlistat on TRAIL-induced apoptosis by measuring sub- $\mathrm{G}_{1}$ population. The administration of orlistat or recombinant human TRAIL alone only weakly induced apoptosis in DU145 and PC3 cells, while the combination with TRAIL at $30 \mathrm{ng} / \mathrm{ml}$ and orlistat at $50 \mu \mathrm{M}$ or more remarkably induced apoptosis (Fig. 1a). To elucidate whether the enhanced apoptosis was mediated through the death receptor DR5, we employed DR5/Fc chimeric protein binding to TRAIL resulting in prevention of the binding to DR5. As a result, the DR5/Fc chimeric protein blocked the enhanced apoptosis in DU145 and PC3 cells (Fig. 1b). This result suggests that the enhanced apoptosis is mediated through DR5.

We also calculated the combination index (C.I.) to evaluate the combinational effect of TRAIL and orlistat. The C.I. was $<1.0$ in all of the combinations, indicating that the combinational effect of TRAIL and orlistat is synergistic in DU145 and PC3 cells (Fig. 1c).

Orlistat induces expression of DR5 protein and $m R N A$ by enhancing DR5 promoter activity in DU145 and PC3 cells. At first, we examined the effects of orlistat on the expression of DR5 protein in DU145 and PC3 cells. The expression of DR5 protein was upregulated in a dose-dependent manner in DU145 and PC3 cells (Fig. 2a). Next, we examined the effect of orlistat on mRNA expression of DR5 in DU145 and PC3 cells. The 

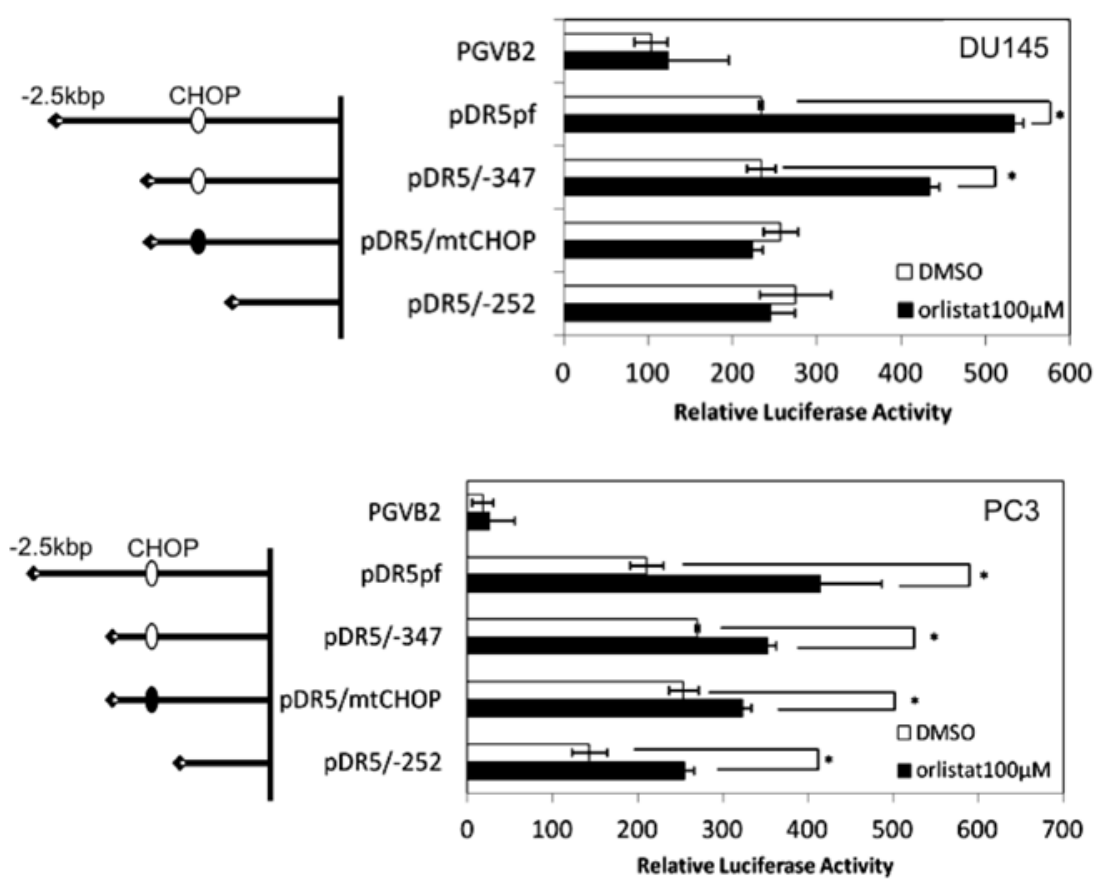

Figure 3. Orlistat enhances the DR5 promoter activity in DU145 and PC3 cells. DU145 or PC3 cells were transiently transfected with the reporter plasmids. After $24 \mathrm{~h}$ of transfection, the cells were treated with $100 \mu \mathrm{M}$ orlistat or DMSO for $24 \mathrm{~h}$, and cell lysates were harvested for luciferase assays as described in Materials and methods. Columns, mean ( $\mathrm{n}=3)$; bars, $\mathrm{SD} .{ }^{* *} \mathrm{P}<0.05$.

DR5 mRNA was also upregulated in a dose-dependent manner in both cell lines (Fig. 2b).

To investigate the mechanism of DR5 induction by orlistat, we carried out a luciferase assay using reporter plasmids containing the DR5 promoter. In both DU145 and PC3 cells, the promoter activity from pDR5PF containing the full-length DR5 promoter was significantly enhanced by orlistat (Fig. 3). Since CHOP upregulates the promoter activity of DR5 through the CHOP binding site $(18,21,22)$, we tried to elucidate whether CHOP was involved in the activation by orlistat. The promoter activity from neither the deleted promoter construct, pDR5/-252, without $\mathrm{CHOP}$ binding site, nor $\mathrm{pDR} 5 / \mathrm{mtCHOP}$ with a mutation in the CHOP-binding site was stimulated by orlistat in DU145 (Fig. 3). In contrast, the promoter activities from both $\mathrm{pDR} 5 /-252$ and pDR5/mtCHOP were still enhanced by orlistat in PC3 cells (Fig. 3). The results suggest that the promoter activity of DR5 in both DU145 and PC3 cells is activated by orlistat, but the activation in DU145 cells is CHOP-dependent and that in PC3 cells is CHOP-independent.

Orlistat induces expression of CHOP protein and $m R N A$ by enhancing CHOP promoter activity in DU145 and PC3 cells. We then examined the effects of orlistat on the expression of CHOP in DU145 and PC3 cells. The expression of CHOP protein was upregulated in a dose-dependent manner in both DU145 and PC3 cells (Fig. 4a), and the CHOP mRNA was also upregulated in a dose-dependent manner in both cell lines (Fig. 4b). In DU145 and PC3 cells, the promoter activities from the fulllength CHOP promoter construct, pCHOP, and the deleted $\mathrm{CHOP}$ promoter construct, $\mathrm{pCHOP} / 150$, were significantly enhanced by orlistat (Fig. 4c). Since endoplasmic reticulum (ER) stress regulates the promoter activity of CHOP through the ER stress responsive element (ERSE) (19), we tried to elucidate whether ER stress was involved in the activation by orlistat. As a result, the promoter activity from pCHOP/-ERSE with a mutation in the ERSE site was not stimulated by orlistat in either cell line (Fig. 4c). The results suggest that orlistat induces ER stress subsequently enhancing the CHOP promoter activity and expression of CHOP mRNA and protein.

CHOP is responsible for the DR5 upregulation in DU145 cells, but not in PC3 cells. We demonstrated that orlistat upregulated the DR5 expression and sensitized malignant tumor cells to TRAIL-induced apoptosis. To confirm whether the upregulation of DR 5 by orlistat is responsible for the sensitization to TRAIL, we performed the knockdown of DR5 expression using siRNA. The treatment with siRNA against DR5 blocked the induction of DR 5 expression by orlistat (Fig. 5a). Furthermore, the knockdown of DR5 also prevented the sensitizing effect of orlistat on TRAIL-induced apoptosis in both cell lines (Fig. 5b).

We then investigated whether CHOP was involved in the sensitization to TRAIL using CHOP siRNA. CHOP siRNA blocked the induction of CHOP expression by orlistat in DU145 and PC3 cells. The suppression of CHOP blocked the induction of the DR5 expression in DU145 cells, but not in PC3 cells (Fig. 5c). Furthermore, the knockdown of CHOP prevented the sensitizing effect of orlistat on TRAIL-induced apoptosis in DU145 cells, but not in PC3 cells (Fig. 5d). These results indicate that there are two different pathways, CHOPdependent and -independent, to induce the DR5 expression by orlistat.

ROS are responsible for the apoptosis induced by the combination of TRAIL and orlistat in DU145 and PC3 cells. It has been reported that ROS production by baicalein causes apoptosis when combined with TRAIL (18). We also found 


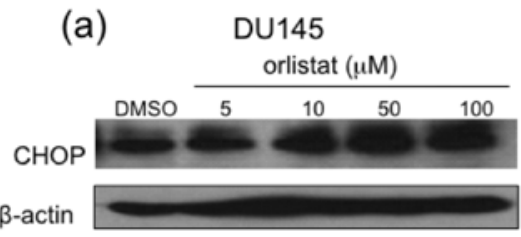

(b)

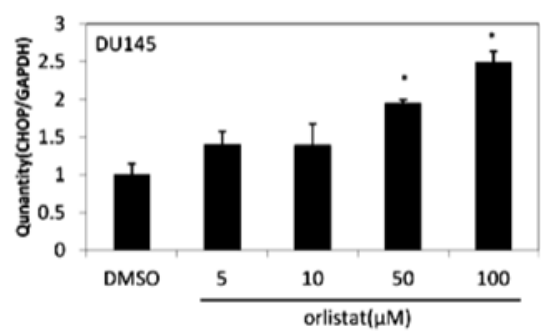

(c)
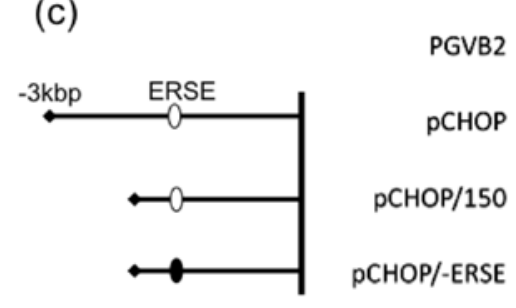

PCHOP/-ERSE

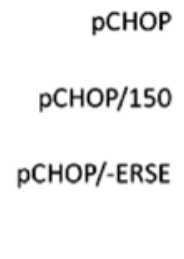

Relative Luciferase Activity
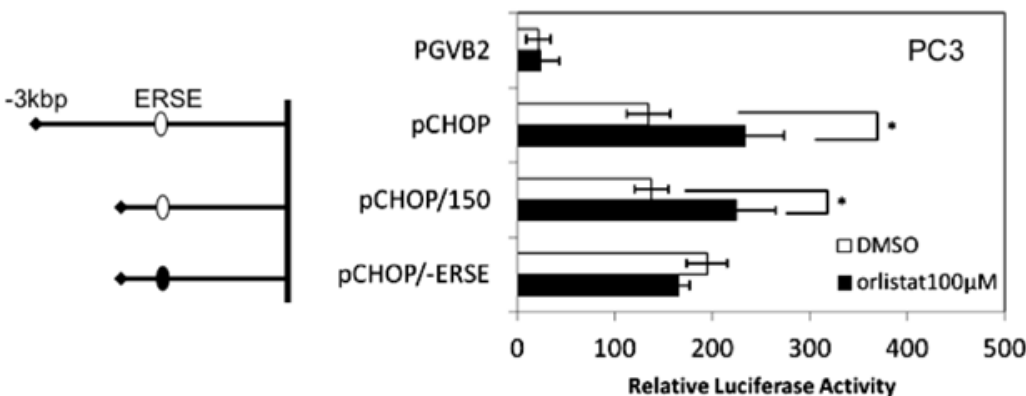

Figure 4. Orlistat induces CHOP protein and mRNA expression through the enhancement of CHOP promoter activity in DU145 and PC3 cells. (a) Whole cell lysates were prepared from DU145 or PC3 cells treated with various concentrations of orlistat for $24 \mathrm{~h}$. The lysates were analyzed by western blotting for CHOP expression. $\beta$-actin was used as a loading control. (b) The induction of CHOP mRNA by orlistat in DU145 or PC 3 cells was analyzed by real-time RT-PCR as described in Materials and methods. Columns, mean $(n=3)$; bars, SD. ${ }^{*} \mathrm{P}<0.05$ compared with the DMSO control. Each sample was standardized by the expression of GAPDH as an internal control. (c) DU145 or PC3 cells were transiently transfected with the reporter plasmids. After $24 \mathrm{~h}$ of transfection, the cells were treated with $100 \mu \mathrm{M}$ orlistat or DMSO for $24 \mathrm{~h}$, and cell lysates were harvested for luciferase assays as described in Materials and methods. Columns, mean (n=3); bars, SD. ${ }^{*} \mathrm{P}<0.05$.

that orlistat could induce intracellular ROS in DU145 and PC3 cells (Fig. 6a and b). A hydrogen peroxide scavenger, catalase, blocked the intracellular ROS induced by orlistat (Fig. 6a and b). Moreover, catalase also blocked the apoptosis induced by the combination of TRAIL and orlistat in both cell lines (Fig. 6c). These findings suggest that the apoptosis induced by the combination of TRAIL and orlistat is dependent on the ROS production by orlistat.

The combination with TRAIL and orlistat does not induce apoptosis in normal peripheral blood mononuclear cells (PBMC) and normal human prostate epithelial cells (PrEC). We investigated whether the combination with TRAIL and orlistat was toxic to normal human cells. We used human PBMC and PrEC as normal models. Neither orlistat nor TRAIL alone induced apoptosis in PBMC and PrEC, and the combination of
TRAIL and orlistat induced hardly any apoptosis in PBMC and PrEC (Fig. 7).

\section{Discussion}

Various chemotherapeutic treatments for HRPC have been proposed, but effective treatments are not established so far. We urgently require effective treatment methods with fewer side effects. Since TRAIL induces apoptosis in HRPC cells, but not in normal cells, the administration of TRAIL is considered one of the promising treatments $(23,24)$. However, since HRPC cells are sometimes resistant to TRAIL, we need to develop a novel strategy to overcome this.

In recent years, anti-hyperlipidemia drugs have been reported to have anti-tumor effects against original expectation (1-3). We tried to examine whether such drugs could 


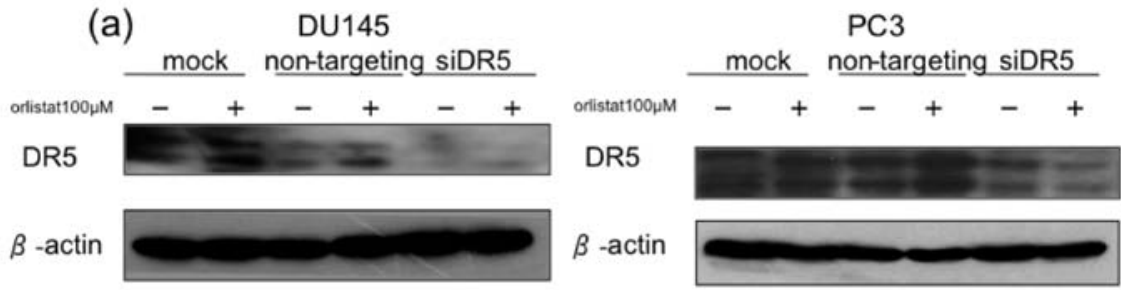

(b) DU145
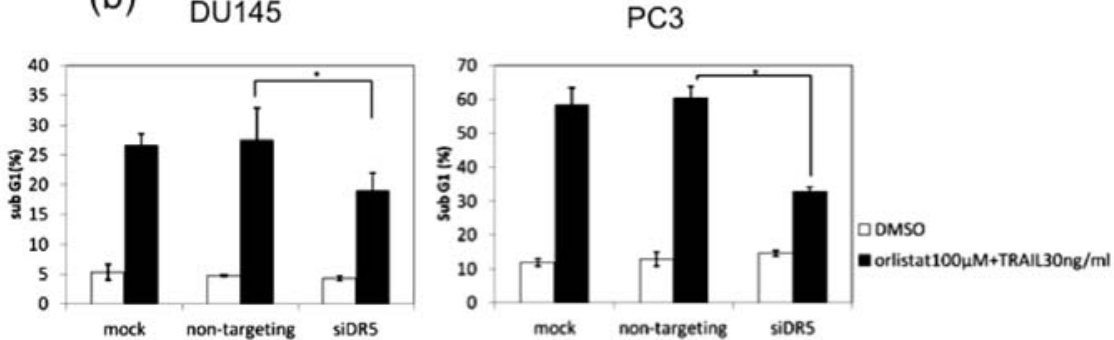

(c)

DU145
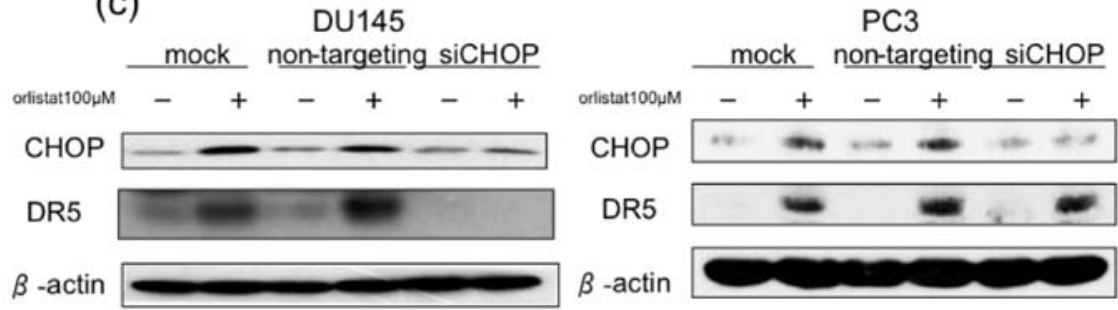

(d)

DU145

$\mathrm{PC} 3$
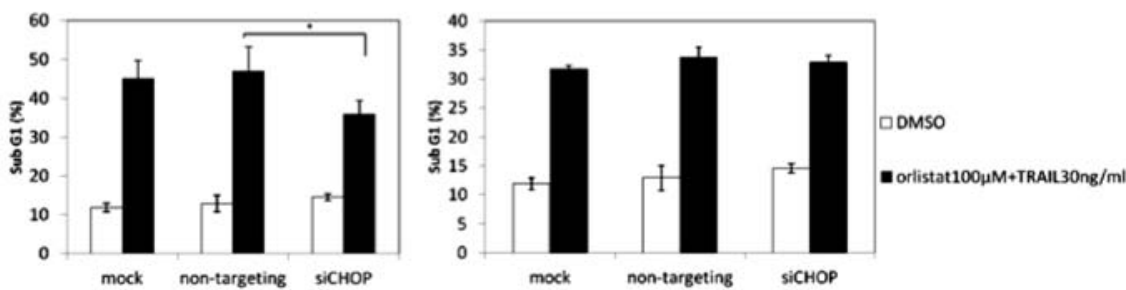

Figure 5. CHOP is induced by orlistat in both DU145 and PC3 cells, however, it is responsible for the DR5 upregulation in DU145 cells, but not in PC3 cells. (a) DU145 or PC3 cells were treated with $100 \mathrm{nM}$ non-targeting or DR5 siRNA. After $24 \mathrm{~h}$ of transfection, cells were treated with $100 \mu \mathrm{M}$ orlistat or DMSO. Cell lysates were analyzed by western blotting for DR5 expression. (b) After $24 \mathrm{~h}$ of transfection, cells were treated with $40 \mu \mathrm{M}$ orlistat and/or $30 \mathrm{ng} / \mathrm{ml}$ of TRAIL. The population of apoptotic cells was analyzed. Columns, means ( $\mathrm{n}=3$ ); bars, SD. " $\mathrm{P}<0.05$. (c) DU145 or PC 3 cells were treated with $100 \mathrm{nM}$ non-targeting or CHOP siRNA. After $24 \mathrm{~h}$ of transfection, cells were treated with $100 \mu \mathrm{M}$ orlistat or DMSO. Cell lysates were analyzed by western blotting for CHOP and DR5 expression. (d) After $24 \mathrm{~h}$ of transfection, cells were treated with $40 \mu \mathrm{M}$ orlistat and/or $30 \mathrm{ng} / \mathrm{ml}$ of TRAIL. The population of apoptotic cells was analyzed. Columns, means $(\mathrm{n}=3)$; bars, $\mathrm{SD} .{ }^{*} \mathrm{P}<0.05$.

increase the sensitivity to TRAIL in HRPC cells. First, we chose orlistat because it has already been used for many years as an anti-obesity drug and its safety has been validated. As demonstrated in the Results, we found a synergistic effect of TRAIL and orlistat in HRPC cells. The combination with orlistat apparently reinforced the apoptosis induced by TRAIL suggesting that orlistat may be useful as a TRAIL sensitizer. Although we have reported on TRAIL sensitivity enhancers $(18-21,25,26)$ other than orlistat, we consider that orlistat is of great interest because it has been proved to be safe for patients.

In addition, we elucidated that the upregulation of DR5 was mediated by enhancement of the promoter activity of DR5 by orlistat. CHOP is a representative transcription factor of DR5 (22), and we examined whether CHOP was involved in the enhancement of the promoter activity by orlistat. The results showed that the activation of the DR5 promoter by orlistat in DU145 cells was CHOP-dependent and that in PC3 cells was CHOP-independent. These results suggest that there are different ways of upregulating DR5 in DU145 cells and PC3 cells, respectively. Orlistat induced the expression of protein and mRNA of CHOP and also enhanced the promoter activity of CHOP in both cell lines. However, the knockdown of CHOP prevented the sensitizing effect of orlistat on TRAIL-induced apoptosis in DU145 cells, but not in PC3 cells. The results also indicate that there are two different pathways, CHOP-dependent and -independent, to induce TRAIL sensitivity by orlistat. Next, we tried to elucidate which molecule was responsible for the induction of CHOP and DR5. It has been reported that ER stress is induced by metabolism medicines (27-29), and we 
(a)
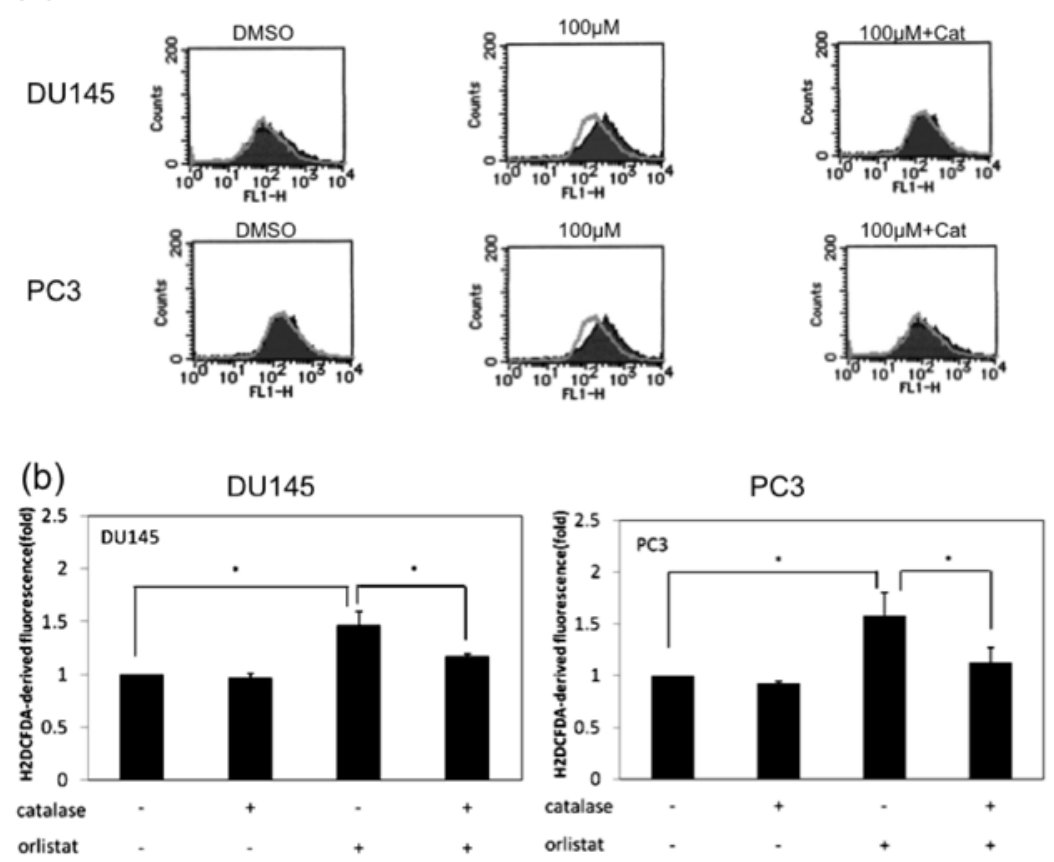

(c)

DU145

PC3

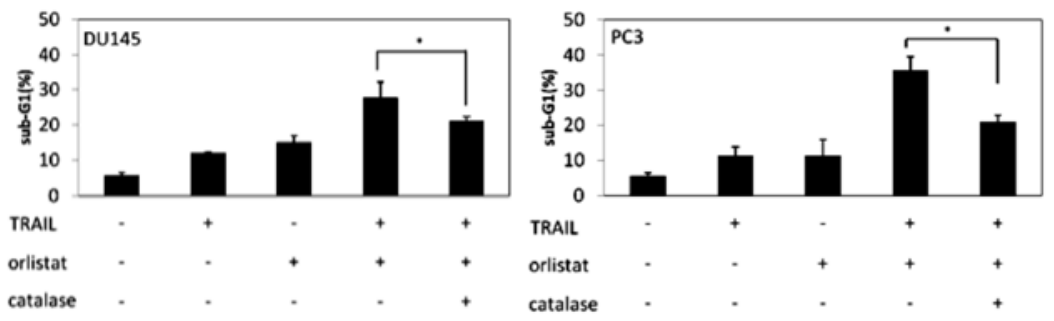

Figure 6. ROS are responsible for the apoptosis induced by the combination of TRAIL and orlistat in both DU145 and PC 3 cells. (a and b) DU145 or PC3 cells were treated with DMSO or $100 \mu \mathrm{M}$ orlistat with or without $1000 \mathrm{U} / \mathrm{ml}$ of catalase. After $24 \mathrm{~h}$ of treatment, ROS levels were analyzed using CM-H $\mathrm{DCF}_{2}$ as described in Materials and methods. Columns, means ( $\mathrm{n}=3$ ); bars, SD. ${ }^{*} \mathrm{P}<0.05$. (c) DU145 or PC3 cells were treated with $100 \mu \mathrm{M}$ orlistat and/or $30 \mathrm{ng} / \mathrm{ml}$ TRAIL with or without $1000 \mathrm{U} / \mathrm{ml}$ catalase. The population of apoptotic cells was analyzed. Columns, mean ( $\mathrm{n}=3$ ); bars, SD. Columns, mean ( $=3$ ); bars, SD. ${ }^{*} \mathrm{P}<0.05$.

PBMC

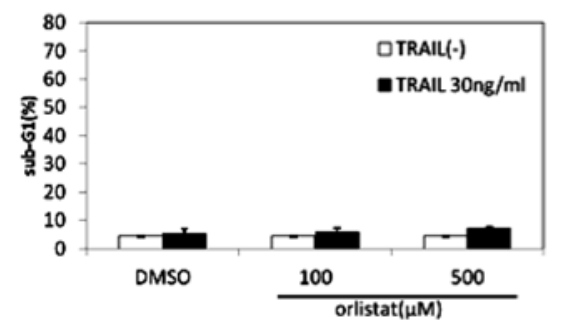

PrEC

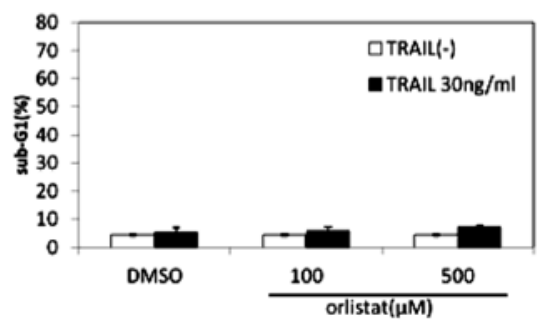

Figure 7. The combination of TRAIL and orlistat does not induce apoptosis to normal PBMC and prostate epithelial cells. PBMC or PrEC were treated with $30 \mathrm{ng} / \mathrm{ml}$ of TRAIL and/or $100 \mu \mathrm{M}$ orlistat for $24 \mathrm{~h}$. The population of apoptotic cells was analyzed. Columns, mean ( $\mathrm{n}=3$ ); bars, SD.

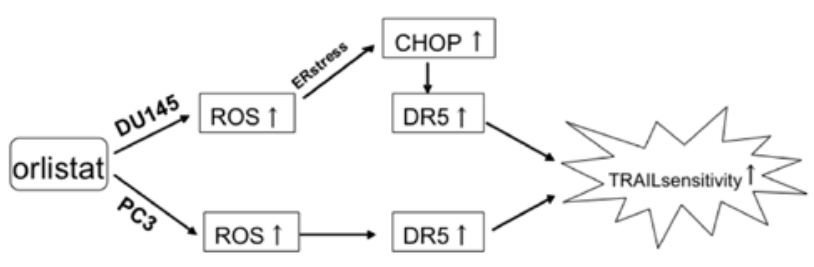

Figure 8. Orlistat has two different pathways to enhance the sensitivity to TRAIL through the upregulation of DR5 in DU145 and PC 3 cells. have also found that orlistat enhanced the promoter activity of CHOP through the ER stress response element. This suggests that orlistat induced ER stress, subsequently inducing CHOP expression. Since it has been reported that ROS induce ER stress (30-32), we investigated whether ROS were involved in the induction of DR5 by orlistat. Orlistat induced intracellular ROS in both DU145 and PC3 cells, and catalase blocked the apoptosis induced by the combination in both cell lines. These 
findings suggest that the apoptosis induced by the combination is dependent on the ROS-producing by orlistat. These results indicate that orlistat acts as a ROS productive reagent.

Recently, it has been reported that several compounds, 15-deoxy- $\Delta^{12,14}$-prostaglandin $\mathrm{J}_{2}$, withaferin $\mathrm{A}$, celastrol, gossypol, dibenzylideneacetone, 5, 7-dimethoxyflavone and cardamonin, induced DR5 expression through the CHOP induction accompanied by ROS generation (33-39). Our data showed that the induction of DR5 by orlistat was also ROS-dependent in both DU145 and PC3 cells. Interestingly, even though the induction in DU145 cells was CHOP-dependent, that in PC3 cells was exceptionally CHOP-independent. The results suggest that there are two different pathways of the upregulation of DR5 by orlistat, which are ROS-CHOP pathway and ROS-direct pathway (Fig. 8).

In conclusion, the administration of TRAIL and orlistat is considered a promising strategy for cancer therapy in HRPC. Moreover, since endogenous TRAIL plays a critical role in immune-surveillance against tumor development, the administration of orlistat may enhance the potency of TRAIL against carcinogenesis, and may become a promising strategy for cancer prevention.

\section{Acknowledgements}

This study was supported by a grant from the Japanese Ministry of Education, Culture, Sports, Science and Technology.

\section{References}

1. Wong WW, Dimitroulakos J, Minden MD and Penn LZ: HMG-CoA reductase inhibitors and the malignant cell: the statin family of drugs as triggers of tumor-specific apoptosis. Leukemia 16: 508-519, 2002.

2. Kawata S, Yamasaki E and Nagase T, et al: Effect of pravastatin on survival in patients with advanced hepatocellular carcinoma. A randomized controlled trial. Br J Cancer 84: 886-891, 2001.

3. Sassano A and Platanias LC: Statins in tumor suppression. Cancer Lett 260: 11-19, 2008.

4. Kridel SJ, Axelrod F, Rozenkrantz N and Smith JW: Orlistat is a novel inhibitor of fatty acid synthase with antitumor activity. Cancer Res 64: 2070-2075, 2004.

5. Pizer ES, Jackisch C, Wood FD, Pasternack GR, Davidson NE and Kuhajda FP: Inhibition of fatty acid synthesis induces programmed cell death in human breast cancer cells. Cancer Res 56: 2745-2747, 1996.

6. Menendez JA, Vellon L and Lupu R: Antitumoral actions of the anti-obesity drug orlistat $\left(\right.$ Xenical $^{\mathrm{TM}}$ ) in breast cancer cells: blockade of cell cycle progression, promotion of apoptotic cell death and PEA3-mediated transcriptional repression of Her2/neu (erb B-2) oncogene. Ann Oncol 16: 1253-1267, 2005.

7. Knowles LM, Axelrod F, Browne CD and Smith JW: A fatty acid synthase blockade induces tumor cell-cycle arrest by downregulating Skp2. J Biol Chem 279: 30540-30545, 2004.

8. Srivastava RK: TRAIL/Apo-2L: mechanisms and clinical applications in cancer. Neoplasia 3: 535-546, 2001.

9. Walczak H, Miller RE and Ariail K, et al: Tumoricidal activity of tumor necrosis factor-related apoptosis-inducing ligand in vivo. Nat Med 5: 157-163, 1999.

10. Takeda K, Smyth MJ, Cretney E, Hayakawa Y, Kayagaki N, Yagita $\mathrm{H}$ and Okumura $\mathrm{K}$ : Critical role for tumor necrosis factor-related apoptosis-inducing ligand in immune surveillance against tumor development. J Exp Med 2: 161-169, 2002.

11. Ichikawa K, Liu W and Zhao L, et al: Tumoricidal activity of a novel anti-human DR5 monoclonal antibody without hepatocyte cytotoxicity. Nat Med 7: 954-960, 2001.

12. Koornstra JJ, Kleibeuker JH and van Geelen CM, Rijcken FE, Hollema H, de Vries EG and de Jong S: Expression of TRAIL (TNF-related apoptosis-inducing ligand) and its receptors in normal colonic mucosa, adenomas, and carcinomas. J Pathol 200: 327-335, 2003.
13. Zhang L and Fang B: Mechanisms of resistance to TRAIL induced apoptosis in cancer. Cancer Gene Ther 12: 228-237, 2005.

14. Ozben T: Oxidative stress and apoptosis: impact on cancer therapy. J Pharm Sci 96: 2181-2196, 2007.

15. Pelicano H, Carney D and Huang P: ROS stress in cancer cells and therapeutic implications. Drug Resist Updat 7: 97-110, 2004.

16. Adler V, Yin Z, Tew KD and Ronai Z: Role of redox potential and reactive oxygen species in stress signaling. Oncogene 18: 6104-6111, 1999.

17. Yoshida T, Maeda A, Tani N and Sakai T: Promoter structure and transcription initiation sites of the human death receptor 5/ TRAIL-R2 gene. FEBS Lett 507: 381-385, 2001.

18. Taniguchi H, Yoshida $\mathrm{T}$ and Horinaka $\mathrm{M}$, et al: Baicalein overcomes tumor necrosis factor-related apoptosis-inducing ligand resistance via two different cell-specific pathways in cancer cells but not in normal cells. Cancer Res 68: 8918-8927, 2008.

19. Saito S, Takahashi S, Takagaki N, Hirose T and Sakai T: 15-Deoxy- $\Delta^{12,14}$-prostaglandin $\mathrm{J}_{2}$ induces apoptosis through activation of the CHOP gene in HeLa cells. Biochem Biophys Res Commun 311: 17-23, 2003.

20. Horinaka M, Yoshida T and Shiraishi T, et al: Luteolin induces apoptosis via death receptor 5 upregulation in human malignant tumor cells. Oncogene 24: 7180-7189, 2005.

21. Yasuda T, Yoshida T and Goda AE, et al: Anti-gout agent allopurinol exerts cytotoxicity to human hormone-refractory prostate cancer cells in combination with tumor necrosis factor-related apoptosis-inducing ligand. Mol Cancer Res 6: 1852-1860, 2008.

22. Yamaguchi $\mathrm{H}$ and Wang HG: $\mathrm{CHOP}$ is involved in endoplasmic reticulum stress-induced apoptosis by enhancing DR 5 expression in human carcinoma cells. J Biol Chem 279: 45495-45502, 2004.

23. Yu R, Mandlekar S, Ruben S, Ni J and Kong AN: Tumor necrosis factor-related apoptosis-inducing ligand-mediated apoptosis in androgen-independent prostate cancer cells. Cancer Res 60: 2384-2389, 2000.

24. Koksal IT, Sanlioglu AD, Kutlu O and Sanlioglu S: Effects of androgen ablation therapy in TRAIL death ligand and its receptors expression in advanced prostate cancer. Urol Int 84: 445-451, 2010.

25. Horinaka M, Yoshida T and Shiraishi T, et al: The combination of TRAIL and luteolin enhances apoptosis in human cervical cancer HeLa cells. Biochem Biophys Res Commun 333: 833-838, 2005.

26. Nakata S, Yoshida T, Horinaka M, Shiraishi T, Wakada M and Sakai T: Histone deacetylase inhibitors upregulate death receptor 5/TRAIL-R2 and sensitize apoptosis induced by TRAIL/APO2-L in human malignant tumor cells. Oncogene 23: 6261-6271, 2004.

27. Carracedo A, Gironella M and Lorente M, Garcia S, Guzman M, Velasco G and Iovanna JL: Cannabinoids induce apoptosis of pancreatic tumor cells via endoplasmic reticulum stress-related genes. Cancer Res 66: 6748-6755, 2006.

28. Senkal CE, Ponnusamy S, Bielawski J, Hannun YA and Ogretmen B: Antiapoptotic roles of ceramide-synthase-6-generated C16-ceramide via selective regulation of the ATF6/CHOP arm of ER-stress-response pathways. FASEB J 24: 296-308, 2010.

29. Fu S, Yang L and Li P, et al: Aberrant lipid metabolism disrupts calcium homeostasis causing liver endoplasmic reticulum stress in obesity. Nature 473: 528-531, 2011.

30. Yokouchi M, Hiramatsu N and Hayakawa K, et al: Involvement of selective reactive oxygen species upstream of proapoptotic branches of unfolded protein response. J Biol Chem 283: 4252-4260, 2008.

31. Fribley A, Zeng Q and Wang CY: Proteasome inhibitor PS-341 induces apoptosis through induction of endoplasmic reticulum stress-reactive oxygen species in head and neck squamous cell carcinoma cells. Mol Cell Biol 24: 9695-9704, 2004

32. Xue X, Piao JH and Nakajima A, et al: Tumor necrosis factor alpha $(\mathrm{TNF} \alpha)$ induces the unfolded protein response (UPR) in a reactive oxygen species (ROS)-dependent fashion, and the UPR counteracts ROS accumulation by TNF $\alpha$. J Biol Chem 280: 33917-33925, 2005.

33. Su RY, Chi KH, Huang DY, Tai MH and Lin WW: 15-Deoxy$\Delta^{12,14}$-prostaglandin $J_{2}$ up-regulates death receptor 5 gene expression in HCT116 cells: involvement of reactive oxygen species and C/EBP homologous transcription factor gene transcription. Mol Cancer Ther 7: 3429-3440, 2008. 
34. Lee TJ, Um HJ, Min DS, Park JW, Choi KS and Kwon TK: Withaferin A sensitizes TRAIL-induced apoptosis through reactive oxygen species-mediated up-regulation of death receptor 5 and down-regulation of c-FLIP. Free Radic Biol Med 46: 1639-1649, 2009.

35. Sung B, Park B, Yadav VR and Aggarwal BB: Celastrol, a triterpene, enhances TRAIL-induced apoptosis through the down-regulation of cell survaival proteins and up-regulation of death receptors. J Biol Chem 285: 11498-11507, 2010.

36. Sung B, Ravindran J, Prasad S, Pandey MK and Aggarwal BB Gossypol induces death receptor-5 through activation of the ROS-ERK-CHOP pathway and sensitizes colon cancer cells to TRAIL. J Biol Chem 285: 35418-35427, 2010.

37. Prasad S, Yadav VR, Ravindran J and Aggarwal BB: ROS and CHOP are critical for dibenxylideneacetone to sensitize tumor cells to TRAIL through induction of death receptors and downregulation of cell survival proteins. Cancer Res 71: 538-549, 2011.
38. Yang JF, Cao JG, Tian L and Liu F: 5, 7-Dimethoxyflavone sensitizes TRAIL-induced apoptosis through DR5 upregulation in hepatocellular carcinoma cells. Cancer Chemother Pharmacol: Jun 10, 2011 (Epub ahead of print).

39. Yadav VR, Prasad S and Aggrwal BB: Cardomonin sensitizes tumor cells to TRAIL through ROS- and CHOP-mediated upregulation of death receptors and downregulation of survival proteins. Br J Pharmacol: Jul 28, 2011 (Epub ahead of print). doi: 10.1111/j.1476-5381.2011.01603.x. 